\title{
A New Algorithm for the End-to-End Delay Bound Based on Statistical Network Calculus
}

\author{
Y.Z. Zhao \\ Department of Electronics \\ Engineering University of CAPF \\ Shaanxi Xi'an, China
}

\begin{abstract}
In order to analyze the delay performance of aggregate traffic, this paper proposes a new algorithm for the End-To-End statistical delay bound of Converged Network with aggregate flows using the theorem of the moment generating function. The results show the algorithm has more Effectiveness and Superiority, it could provide a design reference for the performance evaluation.
\end{abstract}

Keywords-aggregate traffic; statistical network calculus; moment generating function

\section{INTRODUCTION}

With the development of sensors, computers, network communication technology, grid network ${ }^{[1]}$ came into the information technology as a solution to the complex problem of heterogeneous distributed generation technology, the main idea is to form a network of various autonomous section of the main idea is to form a variety of loosely coupled network of loosely coupled autonomous nodes to achieve a large scale multi-type resource sharing and collaborative work, which uses distributed computing services technology, based on a hierarchical system of technical standards, relying on highspeed broadband network, the information system of nodes interconnected by way of information, sharing of resources and collaborative work environment for information to form a grid-based information-sharing networks.

Currently, the army is building a grid of information network is a kind of information service network system of our army and all military information systems Effective integration, providing data, image and voice services for integrated command platform of integrated services capabilities, is to win the effective protection under conditions of future information war. Because of the limited diversity of the grid network service bearer and network resources, Making resource allocation network status significantly more important, therefore, according to the business end QoS indicators, how to reasonable and efficient allocation of network resources has become a focus of the study. To solve this problem, we take advantage of today's popular networkend performance analysis theory - statistical network calculus theoretical modeling and resource allocation based on the specific allocation policy.

\section{THEORETICAL KNOWLEDGE}

Here are the basics of this article needs a detailed description of reference [2]

\section{A. Definition 1. (Fractional Brownian Motion)}

If you reach the traffic $A(t)$ to meet

$$
A(t)=\lambda t+\sigma B(t)
$$

Called self-similar traffic $A(t)$ for fractional Brownian motion (FBM, Fractional Brownian Motion), which $\lambda$ is the average arrival rate of flow, $B(t)$ is usually distributed zero mean, standard self-similar fractal Brownian motion parameters $H \in(0.5,1)$ for the standard deviation $\sigma$. When independent and have the same amount of traffic parameters multiplexing, aggregation streams meet $\lambda_{c o n}=\sum_{i=1}^{m} \lambda_{i}$, $\sigma_{c o n}^{2}=\sum_{i=1}^{m} \sigma_{i}^{2}$, which is the number of the same kinds of polymeric micro business flow.

\section{B. Definition 2. (Statistical Delay Upper Bound)}

$\varepsilon$ is violation of statistical probability under the precondition, the service traffic in the tandem node system delay satisfies

$$
P\left\{W(t) \leq d_{\max }\right\} \geq 1-\varepsilon
$$

\section{Definition3. (Effective Bandwidth)}

Effective bandwidth of the network link bandwidth inherent in unused, usually a measure of network conditions. For traffic, its effective bandwidth $\omega(\theta, t)$ can be defined as

$$
\omega(\theta, t)=\sup _{s \geq 0}\left\{\frac{1}{\theta t} \log E\left[e^{\theta(A(t+s)-A(s))}\right]\right\}
$$

$\theta$ is used to describe the distribution of traffic flows arrive; $\tau$ is used to indicate the length of time intervals.

\section{Definition4. (Moment Generating Function)}

The moment generating function can completely characterize distribution of the random variable $X$, so the 
moment generating function of the random variable become an important research tool. If the expected value of a random variable is a function of the presence, called $e^{\theta x}$ is the moment generating function of the expected value $X$, that is defined as

$$
M_{X}(\theta)=E\left[e^{\theta X}\right]
$$

$E$ is expected, and $\bar{M}_{X}(\theta)=M_{X}(-\theta)=E\left[e^{-\theta X}\right]$.

\section{THE NEW ALGORITHM Is DESCRIBED}

Combined with relevant theory and literature, this paper will present an application of the moment generating function of the new algorithm based on aggregated traffic-end statistical delay bound above. The algorithm is constructed in the form of the moment generating function associated FBM business, not only inherited the ability to use statistical network calculus computing network performance statistics boundaries advantage, consistent FBM in the network selfsimilar characteristics authenticity, better able to flow from the statistical multiplexing Independent Communications the greater the gain, and to effectively improve the resource utilization.

Describe specific steps are as follows:

Step one: Business network packet aggregation streams

If you put some kind of independent business microfluidic FBM after coming together to form a polymeric microfluidic flow for heterogeneous $A_{c o n}=\sum_{i=1}^{m} A_{i}$. In order to simplify and facilitate the presentation $H$ of the analysis, this section considers the same parameters of self-similar traffic aggregation, parameter represents the degree of self-similar traffic bursts, and therefore, by definition 1 and the literature [4] shows that, for an aggregate FBM business envelope can be expressed as

$$
\vartheta_{c o n}(t)=\lambda_{c o n} t+k \sigma_{c o n} t^{H}=\sum_{i=1}^{i=m} \lambda_{i} t+k t^{H} \sqrt{\sum_{i=1}^{i=m} \sigma_{i}^{2}}
$$

where, $m$ is the number of the same kind of service in the microfluidic polymerization.

Step two: Based on the arrival and service curve envelope moment generating function

Combined with moment generating function is defined according to the literature [5] Equation (41), based on aggregate to reach the moment generating function of the envelope $\vartheta_{\text {con }}(t)$,

$M_{\vartheta_{c o n}}(\theta, t)=E\left[e^{\theta \vartheta_{c o n}(t)}\right]=\exp \left\{\lambda_{c o n} \theta t+\frac{\theta^{2} \lambda_{c o n}^{2} \sigma_{c o n}^{2}}{2} t^{2 H}\right\}$

According to the literature [5] Equation (62), based on the service curve moment generating function

$$
M_{S}(-\theta, t)=E\left[e^{-\theta S(t)}\right]=\exp \{-R \theta(t-T)\}
$$

$T$ is the waiting time for the service.

Step three:The upper bound of the probability distribution of the statistical delay

Definition 2 and Chelsea combined delay Chizhov boundary theory can be

$$
\begin{aligned}
P\left\{W^{E 2 E}(t) \leq d_{M G F}^{E 2 E}\right\} & \geq P\left\{\sup _{0 \leq s \leq t}\left[\vartheta^{E 2 E}(s)-\varsigma^{E 2 E}\left(s+d_{M G F}^{E 2 E}\right)\right]>0\right\} \\
& \geq \sum_{s=0}^{t} M_{\vartheta^{E 2 E}(s)}(\theta) M_{\varsigma^{E 2 E}\left(s+d_{M G F}^{E 2 E}\right)}(-\theta) \\
= & {\left[M_{\varsigma^{E 2 E}}(-\theta) \circ M_{\vartheta^{E 2 E}}(\theta)\right]\left(t, d_{M G F}^{E 2 E}\right) }
\end{aligned}
$$

Equation (9) and (10) (11), the final calculation can be obtained through the network end to end delay of string type into the equation in the form of the moment generating function

$$
\begin{gathered}
P\left\{W^{E 2 E}(t) \leq d_{M G F}^{E 2 E}\right\} \geq \exp \left\{\lambda_{c o n} \theta\left(d_{M G F}^{E 2 E}+N T\right)+\frac{\theta^{2} \lambda_{c o n}^{2} \sigma_{c o n}^{2}}{2}\left(d_{M G F}^{E 2 E}+N T\right)^{2 H}\right\} \\
\cdot\left(\frac{1-\exp \left\{-\theta \lambda_{c o n}(2 T+1)\right\}}{1-\exp \left\{-\theta \lambda_{c o n} T\right\}}+\frac{1-\exp \left\{-\theta^{2} \lambda_{c o n}^{2} \sigma_{c o n}^{2}(2 T+1)^{2 H}\right\}}{1-\exp \left\{-\theta^{2} \lambda_{c o n}^{2} \sigma_{c o n}^{2} T^{2 H} / 2\right\}}\right. \\
\left.+\frac{1-\exp \{\theta R(2 T+1)\}}{1-\exp \{\theta R T\}}\right)^{N}
\end{gathered}
$$

Step Four: Statistical delay upper bound expressions

With Combining Definition 3 , order $P\left\{W^{E 2 E}(t) \leq d_{M G F}^{E 2 E}\right\}=1-\varepsilon$, after finishing contain implicit available

$$
d_{M G F}^{E 2 E}=\frac{N \ln \gamma-\ln \varepsilon}{\lambda_{q, c o n} \theta}+\frac{\theta \lambda_{q, c o n}}{2}\left(N T-d_{M G F}^{E 2 E}\right)^{2 H}+N T
$$

Taylor will be in accordance with the formula $\left(N T-d_{M G F}^{E 2 E}\right)^{2 H}$ for the last one . The (14) into (13), through the operation finally get on the moment generating function in the form of end statistical delay bound, namely

$$
d_{M G F}^{E 2 E}=\frac{2 N\left(\ln \gamma+T \lambda_{c o n} \theta\right)-2 \ln \varepsilon+(N T)^{2 H} \theta^{2} \lambda_{c o n}^{2}}{2+2 \theta^{2} \lambda_{c o n}^{2}(N T)^{2 H-1} H}
$$

Among them,

$$
\gamma=\frac{1-\exp \left\{-\theta \lambda_{c o n}(2 T+1)\right\}}{1-\exp \left\{-\theta \lambda_{c o n} T\right\}}+\frac{1-\exp \left\{-\theta^{2} \lambda_{c o n}^{2} \sigma_{c o n}^{2}(2 T+1)^{2 H}\right\}}{1-\exp \left\{-\theta^{2} \lambda_{c o n}^{2} \sigma_{c o n}^{2} T^{2 H} / 2\right\}}
$$




$$
+\frac{1-\exp \{\theta R(2 T+1)\}}{1-\exp \{\theta R T\}}
$$

Thus, the algorithm ends.

Therefore, the above is a new algorithm for solving the delay aggregation traffic converged network end to end.

\section{PERFORMANCE ANALYSIS AND SimULATION COMPARISON}

In this section, we are using Matlab mathematical simulation tools for the last section of the upper bound of the network end to end delay statistics and numerical simulation analysis performed to confirm the validity of the algorithm. In the simulation experiments, set the wait time $T=1 m s$, free parameters $\theta=10^{4}$.

Fig.1 shows the statistical type delay upper bound, contrary to probability and the number of nodes, respectively, in the number of micro-flow relationship is $1,2,5$ case. Set aggregation arrival rate $\lambda_{\text {con }}=0.5 \mathrm{Mbps}$, system service rate $R=100 \mathrm{Mbps}$, aggregation standard deviation $\sigma_{c o n}=25 \mathrm{~kb}$, $H=0.8$. As can be seen from the figure, in general, the end to end delay $d_{M G F}^{E 2 E}$ on the statistical community as contrary to the business community to increase the probability $\varepsilon$ of delay decreases, with the increase of the number $N$ of nodes increases. Under various conditions, with the increase of the number $m$ of micro-flow is increased, and when $\varepsilon$ are small and $d_{M G F}^{E 2 E}$ decrease rapidly with the increase, with $\varepsilon$ increase gradually becomes gentle.

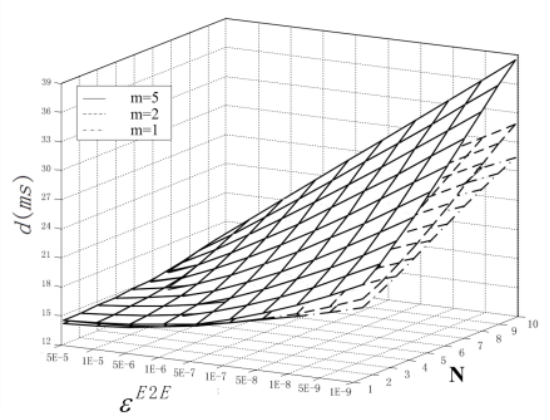

\section{FIGURE I. NETWORK LATENCY STATISTICS ON COMMUNITY AND CONTRARY TO PROBABILITY}

Fig. 2 shows the actual simulation, [3] and the upper bound of the delay comparison service microfluidic number $N=5$ of algorithms and the algorithm in the case of the number of nodes. The figure $d_{\text {sim }}$ represents the actual value of the parameter settings under the same conditions in OPNET simulation results, ${ }_{\text {wen }}$ showing the literature [3] algorithm derived estimates, ${ }_{m y}$ estimates indicate the application of this article or algorithms. Setting the arrival rate $\lambda=1.5 \mathrm{Mbps}$, the standard deviation coefficient $\sigma=75 \mathrm{kp}$, system service rate $R=100 \mathrm{Mbps}$, self-similar parameters $H=0.8$ obtained results shown in Figure 3. The figure shows: the upper bound of the delay and the actual simulation algorithms under two trends are consistent with the increase in both the number of users increases access, when the number $m<20$ of microfluidic too, little difference between the two algorithms, they are closed to $d_{\text {sim }}$; When the number $20<m<80$ of business streams, ${ }^{d}$ usually each node the maximum number of the traffic does not exceed $R / \lambda$, along with the increase $m$, end to end delay after statistical multiplexing is significantly less than before reuse, ${ }^{d m y}$ is the more and more obvious superiority of the algorithm.

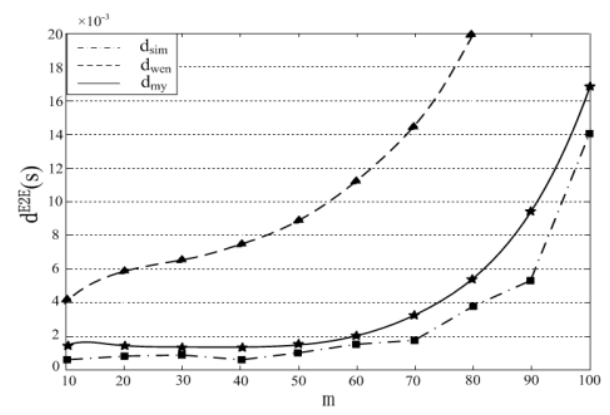

FIGURE II. RELATIONSHIPS AND COMPARISON OF DELAY UPPER BOUND, THE NUMBER OF TRAFFIC

Fig. 3 is a flow of operations in the polymerization in $H=0.65$ and $H=0.85$ of the merits of the algorithm, for convenience, the same 10 microflow taken arrival rate $\lambda_{\text {con }}=1.5 \mathrm{Mbps}, \varepsilon=10^{-9}$. From the figure, in general, the larger the network the more performance $H$ is always poor, delay upper bound is significantly less than the single aggregated traffic stream, and more close to the actual value, but for a single microfluidic higher speed networks, the impact on the network is relatively complex, the magnitude of difference between the larger and the actual value. The curves show that the algorithm of this paper, has obvious advantages in multimicro-flow business.

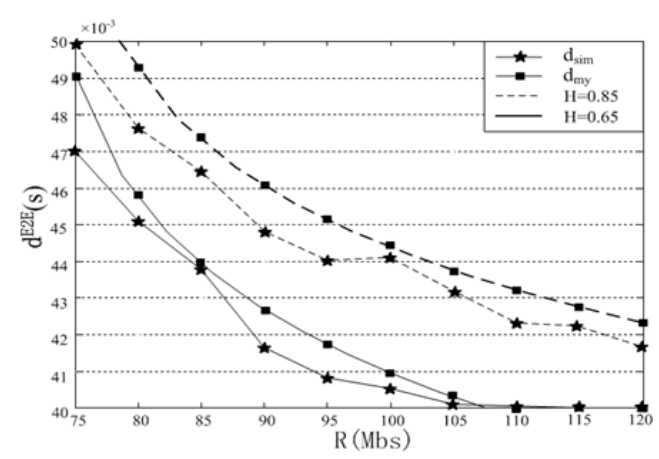

FIGURE III. RELATIONSHIPS AND COMPARISON OF DELAY UPPER BOUND, SYSTEM SERVICE RATE

\section{CONCLUSION}

Firstly, the theoretical minimum plus algebra statistical network calculus basics briefly described, after statistical network calculus model proposed by traditional network calculus theory, self-similar traffic and moment generating functions combined again on the network delay bound 
deduced the moment generating function of the form generated probability model. The model can effectively use statistical independence theories and traditional network Calculus delay estimation, direct convolution avoid complex arithmetic formula, independent of configuration parameters for different node provides a convenient, more accurate characterization of the delay boundary changes. Numerical and simulation results show that the proposed model-end statistical delay upper bound moment generating function of performance evaluation based on FBM business has better adaptability, and provide services to ensure the routing, resource efficient bandwidth allocation control has certain reference value. Therefore, the current real-time QoS guarantee a certain theoretical and practical significance related topics in performance evaluation studies.

\section{REFERENCES}

[1] FOSTER I, KESSELMAN C, T UECKE S. The anatomy of the grid: enabling scalable virtual organization [J]. The International Journal of High Performance Computing Applications, 2001, 15 (3) : 200-222.

[2] R.L.CRUZ. A calculus for network delay, part I and II[J]. IEEE Transactions on Information Theory, 1991,37(1):114-141

[3] I.S. Jacobs and C.P. Bean, "Fine particles, thin films and exchange anisotropy," in Magnetism, vol. III, G.T. Rado and H. Suhl, Eds. New York: Academic, 1963, pp. 271-350.

[4] K. Elissa, "Title of paper if known," unpublished.

[5] R. Nicole, "Title of paper with only first word capitalized," J. Name Stand. Abbrev., in press.

[6] Y. Yorozu, M. Hirano, K. Oka, and Y. Tagawa, "Electron spectroscopy studies on magneto-optical media and plastic substrate interface," IEEE Transl. J. Magn. Japan, vol. 2, pp. 740-741, August 1987 [Digests 9th Annual Conf. Magnetics Japan, p. 301, 1982].

[7] M. Young, The Technical Writer's Handbook. Mill Valley, CA: University Science, 1989. 